Supplementary Experimental Information.

\title{
Regiocontrol in the Oxidative Radical Fragmentation of Benzilidene Acetals and its Mechanistic Implications
}

James McNulty, Jeff Wilson, Amanda C. Rochon

\section{Contents:}

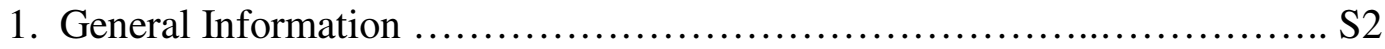

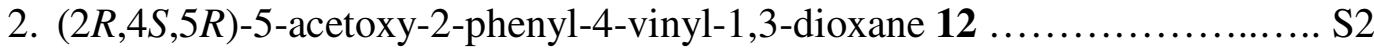

3. (2S,3E)-2-acetyloxy-5-bromo-3-pentenyl benzoate $13 \ldots \ldots \ldots \ldots \ldots \ldots . . \ldots \ldots$

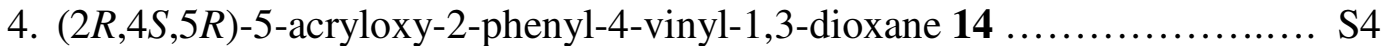

5. (2S,3E)-2-(acryloyloxy)-5-bromo-3-pentenyl benzoate $15 \ldots \ldots \ldots \ldots \ldots . . . . . . .55$

6. (2E,2' $\left.R, 4^{\prime} S, 5^{\prime} R\right)$ Methyl-3-[5'-acetyloxy-2'-phenyl-1',3'-dioxan-4'-yl]-2-

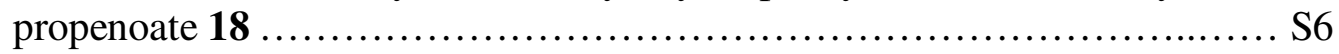

7. $(2 R, 3 R, 4 E)$-2-(acetyloxy)-3-bromo-6-methoxy-6-oxo-4-hexenyl benzoate $22 \mathrm{~S} 7$

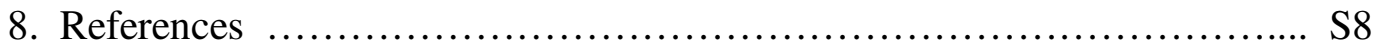

9. ${ }^{1} \mathrm{H}$ and ${ }^{13} \mathrm{C}-\mathrm{NMR}$ spectra for compounds $12, \mathbf{1 3}, \mathbf{1 4}, \mathbf{1 5}, \mathbf{1 8}$ and 22 .... S9-S20 


\section{General:}

Pyridine was distilled over calcium hydride and THF and toluene dried over sodium using benzophenone indicator. Benzoyl peroxide (75\% purity) was dried in small batches (10-30 mg) before using under high vacuum at $50^{\circ} \mathrm{C}$ for at least 1.5 hour. Reactions were performed in oven-dried glass vessels; thermal reactions were conducted in a thermostat controlled oil bath. ${ }^{1} \mathrm{H}-\mathrm{NMR},{ }^{13} \mathrm{C}-\mathrm{NMR}$ were recorded at 300 and $75 \mathrm{MHz}$ respectively in $\mathrm{CDCl}_{3}$.

$\underline{(2 R, 4 S, 5 R)-5 \text {-acetoxy-2-phenyl-4-vinyl-1,3-dioxane } \mathbf{1 2}}$.

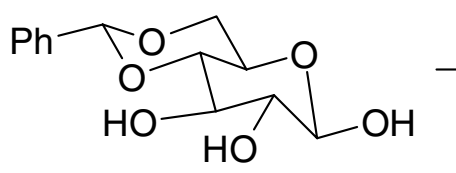

A

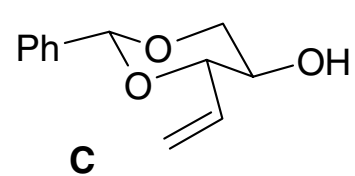

Ref. 1

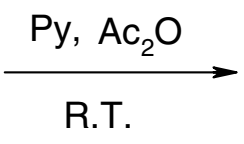

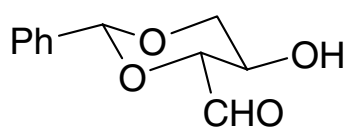

B

Ref. 2
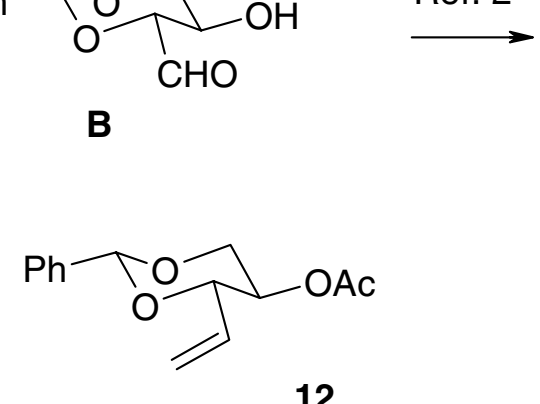

Compound 12 was prepared from D-glucose via its 4,6-benzilidene acetal A through known aldehyde $\mathbf{B}^{1}$ and olefin $\mathbf{C .}^{2}$ To a round bottom flask was added 2-phenyl-4-vinyl1,3-dioxan-5-ol ${ }^{1} \mathbf{C}(50.0 \mathrm{mg}, 0.243 \mathrm{mmol})$ followed by pyridine $(1.0 \mathrm{~mL})$ and acetic anhydride $(1.0 \mathrm{~mL})$. The solution was stirred at room temperature under argon for $2 \mathrm{~h}$ when the volatiles were removed in-vacuo through a dry-ice trap. The residue was partitioned between dichloromethane $(3 \times 10 \mathrm{~mL})$ and $5 \%$ aqueous sodium bicarbonate. The organic phase was dried $\left(\mathrm{Na}_{2} \mathrm{SO}_{4}\right)$, filtered and concentrated on the rotary evaporator to give 12 as a colorless crystalline mass in quantitative yield. M.p. $60-65{ }^{\circ} \mathrm{C} ;{ }^{1} \mathrm{H}$ NMR 
$\left(300 \mathrm{MHz}, \mathrm{CDCl}_{3}\right) \delta 2.01(\mathrm{~s}, 3 \mathrm{H}), 3.70(\mathrm{t}, 2 \mathrm{H}, 10.4 \mathrm{~Hz}), 4.23(\mathrm{dd}, 1 \mathrm{H}, \mathrm{J}=6.6,9.5 \mathrm{~Hz}), 4.41$

(dd, 2H, J=5.3, 10.6 Hz), 4.89 (dt, 1H, J=5.3, 9.9 Hz), 5.30 (d, 1H, J=10.5 Hz), 5.44 (d, $1 \mathrm{H}, \mathrm{J}=17.1 \mathrm{~Hz}), 5.58(\mathrm{~s}, 1 \mathrm{H}), 5.95-5.87(\mathrm{~m}, 1 \mathrm{H}), 7.38(\mathrm{~m}, 3 \mathrm{H}), 7.51(\mathrm{~m}, 2 \mathrm{H}) ;{ }^{13} \mathrm{C} \mathrm{NMR}$ $\left(300 \mathrm{MHz}, \mathrm{CDCl}_{3}\right) \delta 169.3,137.7,134.4,129.5,128.7,126.6,119.3,101.6,80.76,68.55$, 66.6, 21.2; IR (KBr film) 3466, 2880, 2854, 1738, 1454, 1376, 1254, 1113, 1087, 1053, 1030, 994, 764, $700 \mathrm{~cm}^{-1}$; MS (EI) m/z 43 (100\%), 149 (52), 107 (42), 91 (20), 77 (14), 51(7), 205 (4), 248 (2), 188 (2), 171 (1); ); HRMS (EI) m/z calcd. for $\mathrm{C}_{14} \mathrm{H}_{16} \mathrm{O}_{4}$ 248.1049, found 248.1037; $[\alpha]_{\mathrm{D}}-42.71^{\circ}(\mathrm{c}=0.10, \mathrm{MeOH})$.

(2S,3E)-2-acetyloxy-5-bromo-3-pentenyl benzoate $\mathbf{1 3}$.

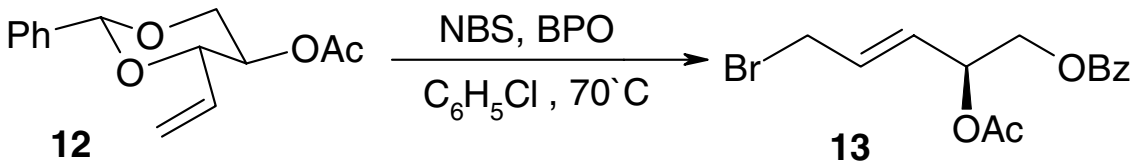

To a round bottom flask charged with compound $12(10.0 \mathrm{mg}, 0.040 \mathrm{mmol})$ was added NBS (8.6 mg, $0.048 \mathrm{mmol})$, BPO (1.0 mg, $0.002 \mathrm{mmol}$ ), and $1.0 \mathrm{~mL}$ of $\mathrm{PhCl}$ under argon. The flask was fitted with a condenser and placed in an oil bath pre-heated at $70^{\circ} \mathrm{C}$. After $1 \mathrm{~h}$ a second addition of NBS (1.4 mg, $0.008 \mathrm{mmol})$ and BPO (1.0 mg, $0.002 \mathrm{mmol})$ was added in $100 \mu \mathrm{L}$ of $\mathrm{PhCl}$. After stirring at $70^{\circ} \mathrm{C}$ for a further $2 \mathrm{~h}$ the reaction was cooled to room temp. and diluted with $5 \% \mathrm{NaHCO}_{3}(\mathrm{aq})$, followed by work up from dichloromethane as described for $\mathbf{1 2}$. The crude product was purified over silica ( $30 \mathrm{x} 1$ $\mathrm{cm}$ column) eluting with 95:5 hexane:ethyl acetate. Compound $\mathbf{1 3}$ was isolated as a clear colorless oil in $64.2 \%$ yield along with $21.5 \%$ recovery of starting material $\mathbf{1 2}$. Compound 13 had ${ }^{1} \mathrm{H}$ NMR $\left(300 \mathrm{MHz}, \mathrm{CDCl}_{3}\right) \delta 2.12(\mathrm{~s}, 3 \mathrm{H}), 3.97(\mathrm{~d}, 1 \mathrm{H}, \mathrm{J}=7.4 \mathrm{~Hz})$, 
$4.38(\mathrm{dd}, 1 \mathrm{H}, \mathrm{J}=6.6,11.8 \mathrm{~Hz}), 4.49$ (dd, 1H, J=4.0, $11.8 \mathrm{~Hz}), 5.70$ (dd, 1H, J=6.3, 10.5 Hz), $5.84(\mathrm{dd}, 1 \mathrm{H}, \mathrm{J}=6.4,15.3), 6.10(\mathrm{~m}, 1 \mathrm{H}), 7.38(\mathrm{t}, 2 \mathrm{H}, \mathrm{J}=7.6 \mathrm{~Hz}), 7.49(\mathrm{t}, 1 \mathrm{H}, \mathrm{J}=7.4$ $\mathrm{Hz}), 7.95(\mathrm{~d}, 2 \mathrm{H}, \mathrm{J}=7.2 \mathrm{~Hz}) ;{ }^{13} \mathrm{C} \mathrm{NMR}\left(300 \mathrm{MHz}, \mathrm{CDCl}_{3}\right) \delta 170.3,166.5,133.6,131.1$, 130.1, 129.3, 128.8, 126.7, 71.1, 65.3, 31.3, 21.4; IR (KBr film) 3441, 2956, 2924, 1720, 1646, 1451, 1372, 1273, 1228, 1110, 1025, $711 \mathrm{~cm}^{-1}$; MS (EI) m/z 105 (100\%), 43 (38), 77 (28), 149 (14), 247 (10), 122 (9), 177 (4); HRMS (EI) m/z calcd. for $\mathrm{C}_{14} \mathrm{H}_{14} \mathrm{O}_{4}{ }^{79} \mathrm{Br}$ $325.0075\left(\mathrm{M}^{+}-\mathrm{H}\right)$, found $325.0070 ;[\alpha]_{\mathrm{D}}+30.8^{\circ}(\mathrm{c}=0.10, \mathrm{MeOH})$.

$\underline{(2 R, 4 S, 5 R)-5 \text {-acryloxy-2-phenyl-4-vinyl-1,3-dioxane } \mathbf{1 4}}$.

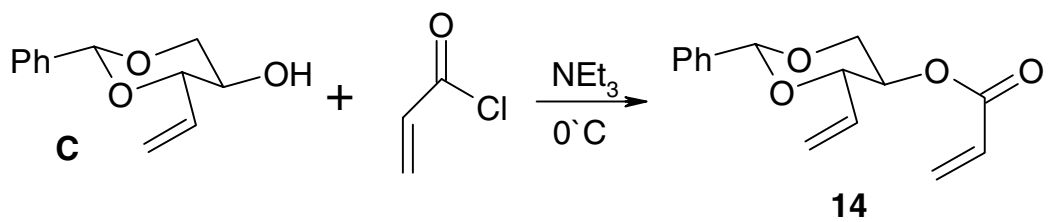

A dry round bottom flask was charged with 2-phenyl-4-vinyl-1,3-dioxan-5-ol C (50.0 mg, $0.243 \mathrm{mmol}$ ) in $1.0 \mathrm{~mL}$ of DCM and then cooled to $0^{\circ} \mathrm{C}$ under argon. To this was slowly added triethylamine $(29 \mu \mathrm{L}, 0.292 \mathrm{mmol})$ followed by acryloyl chloride $(23.7 \mu \mathrm{L} 0.292 \mathrm{mmol})$. The resulting solution was stirred at $0{ }^{\circ} \mathrm{C}$ for $2.5 \mathrm{~h}$ at which time a second addition of triethylamine and acryloyl chloride (same amounts as above) were added and stirring continued for another $15 \mathrm{~h}$. The reaction mixture was diluted with 5\% sodium bicarbonate (aq) and extracted with dichloromethane (3x15mL). After drying, filtering and solvent removal, the organic residue was purified over silica $(30 \times 1 \mathrm{~cm})$ eluting with the hexanes:ethyl acetate 85:15 (100 mL) with gradient to hexanes:ethyl acetate 75:25. Compound $\mathbf{1 4}$ was isolated in $71 \%$ yield as a clear, slightly yellow oil. ${ }^{1} \mathrm{H}$ NMR (300MHz, $\left.\mathrm{CDCl}_{3}\right) \delta 3.74(\mathrm{t}, 1 \mathrm{H}, \mathrm{J}=10.5 \mathrm{~Hz}), 4.29(\mathrm{dd}, 1 \mathrm{H}, \mathrm{J}=6.4,9.5 \mathrm{~Hz}), 4.46$ 
(dd, 1H, J=5.3, 10.6 Hz), $4.96(d t, 1 H, J=5.3,9.8 \mathrm{~Hz}), 5.30(\mathrm{~d}, 1 \mathrm{H}, \mathrm{J}=10.6 \mathrm{~Hz}), 5.44$ (d, 1H, J=17.2 Hz), 5.60 (s, 1H), $5.91(\mathrm{~m}, 2 \mathrm{H}), 6.12$ (dd, 1H, J=10.5, $17.3 \mathrm{~Hz}), 6.45$ (dd, 1H, 1.3, 17.3 Hz), $7.38(\mathrm{~m}, 3 \mathrm{H}), 7.55(\mathrm{~m}, 2 \mathrm{H}) ;{ }^{13} \mathrm{C} \mathrm{NMR}\left(300 \mathrm{MHz}, \mathrm{CDCl}_{3}\right) \delta$ 164.6, 137.7, $134.2,132.2,129.5,128.7,128.1,126.6,119.3,101.62,80.7,68.6,66.7$; IR (KBr film) 3091, 3041, 2969, 2869, 1728, 1635, 1457, 1404, 1285, 1103, 1024, 1005, 767, $702 \mathrm{~cm}^{-1}$; MS (EI) m/z 55 (100\%), 161 (92), 105 (28), 91 (119), 149 (15), 77 (77), 259 (4), 204 (2), 188 (2), 260 (1); HRMS (EI) m/z calcd. for $\mathrm{C}_{15} \mathrm{H}_{16} \mathrm{O}_{4} 260.1049$, found 260.1033; $[\alpha]_{\mathrm{D}}-$ $55.2^{\circ}(\mathrm{c}=0.2, \mathrm{MeOH})$.

(2S,3E)-2-(acryloyloxy)-5-bromo-3-pentenyl benzoate $\mathbf{1 5}$.

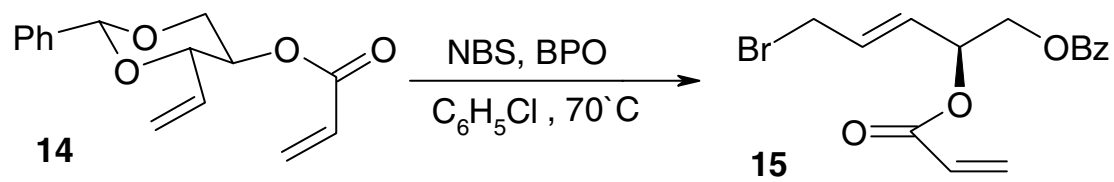

A round bottom flask was charged with compound 14 (8.7 mg, $0.033 \mathrm{mmol})$, NBS (7.1 $\mathrm{mg}, 0.040 \mathrm{mmol})$ and $\mathrm{BPO}(0.6 \mathrm{mg}, 0.002 \mathrm{mmol})$ dissolved in $1.0 \mathrm{~mL}$ of $\mathrm{PhCl}$. The mixture was placed on an oil bath pre-heated at $70^{\circ} \mathrm{C}$. After $1 \mathrm{~h}$, a second addition of NBS (1.4 mg, $0.008 \mathrm{mmol})$ and BPO (0.6 mg, $0.002 \mathrm{mmol})$ was made in $100 \mu \mathrm{L}$ of $\mathrm{PhCl}$.

After stirring at $70^{\circ} \mathrm{C}$ for a further $2 \mathrm{~h}$ the reaction was cooled and partitioned between $5 \%$ $\mathrm{NaHCO}_{3}(\mathrm{aq})$ and dichloromethane $(3 \times 10 \mathrm{~mL})$. The organic phases were dried $\left(\mathrm{Na}_{2} \mathrm{SO}_{4}\right)$, filtered and concentrated to give the crude product that was purified through a silica $(30 \mathrm{x}$ $1 \mathrm{~cm}$ ) column using 60:40 dichloromethane : hexanes as eluant. Product 15 was obtained as a colorless oil in $55.2 \%$ yield along with recovery of $25.3 \%$ starting material 14 .

Compound 15: ${ }^{1} \mathrm{H}$ NMR $\left(300 \mathrm{MHz}, \mathrm{CDCl}_{3}\right) \delta 3.88(\mathrm{~d}, 2 \mathrm{H}, \mathrm{J}=7.3 \mathrm{~Hz}), 4.35(\mathrm{dd}, 1 \mathrm{H}$, 
$\mathrm{J}=11.7,6.6 \mathrm{~Hz}), 4.43(\mathrm{dd}, 1 \mathrm{H}, \mathrm{J}=11.7,4.0 \mathrm{~Hz}), 5.69(\mathrm{~m}, 1 \mathrm{H}), 5.75(\mathrm{~m}, 1 \mathrm{H}), 5.81(\mathrm{~d}, 1 \mathrm{H}$, $\mathrm{J}=9.8 \mathrm{~Hz}), 6.05(\mathrm{~m}, 2 \mathrm{H}), 6.39(\mathrm{~d}, 1 \mathrm{H}, \mathrm{J}=17.3 \mathrm{~Hz}), 7.37(\mathrm{t}, 2 \mathrm{H}, \mathrm{J}=7.6 \mathrm{~Hz}), 7.51(\mathrm{t}, 1 \mathrm{H}$, $\mathrm{J}=7.3), 7.95(\mathrm{~d}, 2 \mathrm{H}, \mathrm{J}=7.3 \mathrm{~Hz}) ;{ }^{13} \mathrm{C} \mathrm{NMR}\left(300 \mathrm{MHz}, \mathrm{CDCl}_{3}\right) \delta 166.5,165.4,133.6$, 132.2, 131.2, 130.1, 129.9, 129.1, 128.8, 128.3, 71.3, 65.3, 31.3; IR (KBr film) 3068, 2924, 2852, 1723, 1272, 1183, 1113, $712 \mathrm{~cm}^{-1}$; LRMS (EI) m/z 105 (100\%), 55 (70), 259 (29), 77 (26), 124 (13), 150 (7), 260 (5), 188 (2); HRMS (EI) m/z calcd. for $\mathrm{C}_{15} \mathrm{H}_{14}{ }^{79}{ }_{\mathrm{Br}} \mathrm{O}_{4}$ (M-H) 337.0075, found 336.9904; $[\alpha]_{\mathrm{D}}+2.3^{\circ}(\mathrm{c}=0.09, \mathrm{MeOH})$.

$\underline{\left(2 E, 2^{\prime} R, 4^{\prime} S, 5^{\prime} R\right) \text { Methyl-3-[5'-acetyloxy-2'-phenyl-1',3'-dioxan-4'-yll-2-propenoate } 18 .}$

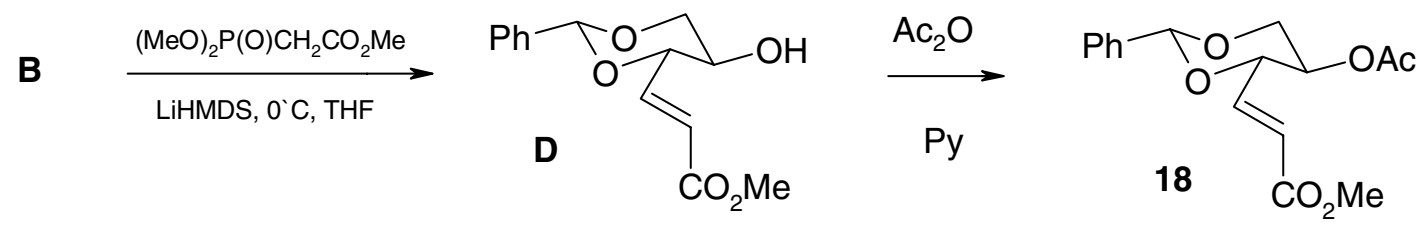

Into a dried round bottom flask was weighed trimethyl phosphonoacetate $(182.9 \mathrm{mg}, 1.00$ mmol). Dry THF ( $3.5 \mathrm{~mL})$ was added and the solution stirred at $0^{\circ} \mathrm{C}$ for 5 min followed by the addition of LiHMDS (1.0 mL, 1.0M in THF, $1.00 \mathrm{mmol})$ and the mixture allowed to stir at $0^{\circ} \mathrm{C}$ for $15 \mathrm{~min}$. At this time a solution of freshly prepared aldehyde B $(69.7 \mathrm{mg}$, $0.335 \mathrm{mmol})$ in dry THF $(1.5 \mathrm{~mL})$ was slowly added via canula under Ar over $\sim 2 \mathrm{~min}$ maintained at $0^{\circ} \mathrm{C}$. After 20 min the reaction was quenched through the addition of saturated aqueous $\mathrm{NH}_{4} \mathrm{Cl}(3 \mathrm{~mL})$ and extraction into ethyl acetate $(3 \times 10 \mathrm{~mL})$. The crude organic residue was purified through a silica gel column $(30 \mathrm{x} 1 \mathrm{~cm})$ using hexanes:ethyl acetate 80:20 slow gradient to 70:30 v/v as eluant. The isolated major $(E)$-isomer $\mathbf{D}$ was isolated as a white solid, $79.5 \mathrm{mg}, 80.7 \%$ yield: m.p. $122-125^{\circ} \mathrm{C} ;{ }^{1} \mathrm{H} \mathrm{NMR}(300 \mathrm{MHz}$, $\left.\mathrm{CDCl}_{3}\right) \delta 3.70(\mathrm{~d}, 2 \mathrm{H}, \mathrm{J}=7.2 \mathrm{~Hz}), 3.78(\mathrm{~s}, 3 \mathrm{H}), 4.27(\mathrm{~m}, 1 \mathrm{H}), 4.35(\mathrm{~d}, 1 \mathrm{H}, \mathrm{J}=5.8 \mathrm{~Hz}), 5.59$ 
(s, 1H), $6.25(\mathrm{dd}, 1 \mathrm{H}, \mathrm{J}=1.3,15.9 \mathrm{~Hz}), 7.22(\mathrm{dd}, 1 \mathrm{H}, \mathrm{J}=4.5,15.9 \mathrm{~Hz}), 7.39(\mathrm{~m}, 3 \mathrm{H}), 7.51$

$(\mathrm{m}, 2 \mathrm{H}) ;{ }^{13} \mathrm{C} \mathrm{NMR}\left(300 \mathrm{MHz}, \mathrm{CDCl}_{3}\right) \delta 143.7,129.5,128.7,126.5,122.6,101.2,80.8$,

71.5, 65.8, 52.2; IR (KBr film) 3439, 2950, 2864, 1709, 1660, 1453, 1319, 1204, 1080, $1021 \mathrm{~cm}^{-1}$; MS (EI) m/z 107 (100\%), 149 (30), 91 (17), 79 (14), 115 (12), 221 (3), 263

(2); HRMS (EI) $\mathrm{m} / \mathrm{z}$ calcd. for $\mathrm{C}_{14} \mathrm{H}_{15} \mathrm{O}_{5} 263.0920\left(\mathrm{M}^{+}-\mathrm{H}\right)$, found 263. 0920; $[\alpha]_{\mathrm{D}}-43.9^{\circ}$ $(\mathrm{c}=0.10, \mathrm{MeOH})$.

To a round bottom flask containing $\mathbf{D}(44.5 \mathrm{mg}, 0.19 \mathrm{mmol})$ was added dry pyridine $(1.0 \mathrm{~mL})$ followed by acetic anhydride $(1.0 \mathrm{~mL})$ under argon. The solution stirred magnetically at room temperature for $2 \mathrm{~h}$ whereupon the volatiles were removed in-vacuo and the residue partitioned between dichloromethane $(15 \mathrm{~mL}$, and $2 \times 10 \mathrm{~mL})$ and $5 \%$ $\mathrm{NaHCO}_{3}$ (aq). Product $\mathbf{1 8}$ was obtained pure by NMR in quantitative yield as a white crystalline solid: m.p. $89-92{ }^{\circ} \mathrm{C} ;{ }^{1} \mathrm{H}$ NMR $\left(300 \mathrm{MHz}, \mathrm{CDCl}_{3}\right) \delta 2.12(\mathrm{~s}, 3 \mathrm{H}), 3.69(\mathrm{t}, 1 \mathrm{H}$, $\mathrm{J}=10.5 \mathrm{~Hz}), 3.77(\mathrm{~s}, 3 \mathrm{H}), 4.47(\mathrm{~m}, 2 \mathrm{H}), 4.80(\mathrm{dt}, 1 \mathrm{H}, \mathrm{J}=10.0,5.2 \mathrm{~Hz}), 5.53(\mathrm{~s}, 1 \mathrm{H}), 6.21$ $(\mathrm{d}, 1 \mathrm{H}, \mathrm{J}=15.8 \mathrm{~Hz}), 6.99(\mathrm{dd}, 1 \mathrm{H}, \mathrm{J}=15.1,4.5 \mathrm{~Hz}), 7.40(\mathrm{~m}, 3 \mathrm{H}), 7.51(\mathrm{~m}, 2 \mathrm{H}) ;{ }^{13} \mathrm{C}$ NMR $\left(300 \mathrm{MHz}, \mathrm{CDCl}_{3}\right) \delta 169.9,166.8,142.4,137.3,129.7,128.7,126.6,123.0,101.6,77.7$, 68.5, 66.6, 52.2, 21.2; IR (KBr film) 3071, 2941, 1724, 1651, 1456, 1384, 1221, 1046 $\mathrm{cm}^{-1}$; MS (EI) m/z 149 (100\%), 43 (100), 107 (58), 169 (10), 305 (3), 264(2), 306 (1); HRMS (EI) m/z calcd. for $\mathrm{C}_{16} \mathrm{H}_{18} \mathrm{O}_{6} 306.1103$, found 306.1102; $[\alpha]_{\mathrm{D}}-38.5^{\circ}(\mathrm{c}=0.10$, $\mathrm{MeOH})$.

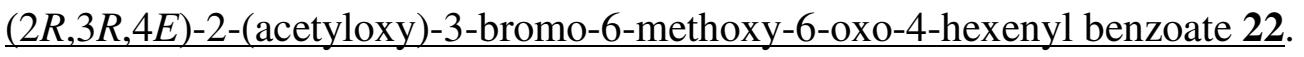



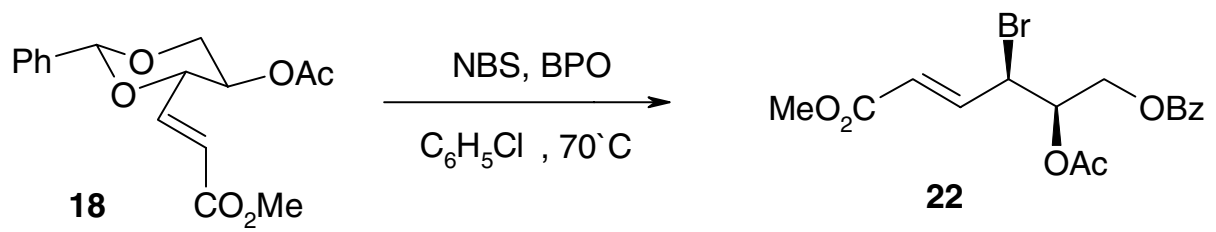

To a dry round bottom flask containing compound $\mathbf{1 8}(33.2 \mathrm{mg}, 0.108 \mathrm{mmol})$ was added NBS (19.2 mg, 0.108), BPO (2.0 mg, $0.006 \mathrm{mmol})$ and $1.0 \mathrm{~mL}$ of $\mathrm{PhCl}$ under argon. The mixture was immersed in a pre-heated oil bath at $70^{\circ} \mathrm{C}$ for $1 \mathrm{~h}$ at which time a second addition of NBS (2.0 mg, $0.011 \mathrm{mmol})$ and BPO $(2.0 \mathrm{mg}, 0.006 \mathrm{mmol})$ was added in 100 $\mu \mathrm{L}$ of $\mathrm{PhCl}$. After stirring at $70^{\circ} \mathrm{C}$ for a further $2 \mathrm{~h}$ the reaction was cooled and partitioned between $5 \% \mathrm{NaHCO}_{3}(\mathrm{aq})$ and dichloromethane $(3 \times 10 \mathrm{~mL})$. The organic phases were dried $\left(\mathrm{Na}_{2} \mathrm{SO}_{4}\right)$, filtered and concentrated to give the crude product that was purified through a silica column $(30 \times 1 \mathrm{~cm})$ using hexanes:ethyl acetate 90:10 v/v, gradient elution to 70:30 hexanes:ethyl acetate as eluant. The product $\mathbf{2 2}$ was isolated as a clolorless oil in $61.0 \%$ yield: ${ }^{1} \mathrm{H}$ NMR $\left(300 \mathrm{MHz}, \mathrm{CDCl}_{3}\right) \delta 2.16(\mathrm{~s}, 3 \mathrm{H}), 3.77(\mathrm{~s}, 3 \mathrm{H})$, $4.44(\mathrm{dd}, 1 \mathrm{H}, \mathrm{J}=11.0,5.8 \mathrm{~Hz}), 4.64(\mathrm{dd}, 1 \mathrm{H}, \mathrm{J}=12.0,4.0 \mathrm{~Hz}), 4.82(\mathrm{dd}, 1 \mathrm{H}, \mathrm{J}=10.1,6.1$ Hz), $5.45(\mathrm{~m}, 1 \mathrm{H}), 6.10(\mathrm{~d}, 1 \mathrm{H}, \mathrm{J}=15.1 \mathrm{~Hz}), 7.02(\mathrm{dd}, 1 \mathrm{H}, \mathrm{J}=9.6,15.3 \mathrm{~Hz}), 7.48(\mathrm{t}, 2 \mathrm{H}$, $\mathrm{J}=7.5 \mathrm{~Hz}), 7.61(\mathrm{t}, 1 \mathrm{H}, \mathrm{J}=7.5 \mathrm{~Hz}), 8.02(\mathrm{~d}, 2 \mathrm{H}, \mathrm{J}=7.2 \mathrm{~Hz}) ;{ }^{13} \mathrm{C} \mathrm{NMR}\left(300 \mathrm{MHz}, \mathrm{CDCl}_{3}\right)$ $\delta 170.1,166.2,165.9,141.7,134.1,133.8,130.1,128.9,125.0,72.4,63.7,52.4,48.8$, 21.1; IR (KBr film) 1724, 1643, 1271, 1222, 1110, 1069, $712 \mathrm{~cm}^{-1}$; MS (EI) m/z 105 (100\%), 43 (31), 77 (18), 305 (13), 220 (6), 384 (1), 386 (1); HRMS (EI) m/z calcd. for $\mathrm{C}_{16} \mathrm{H}_{17} \mathrm{O}_{6}{ }^{79} \mathrm{Br} 384.0209$, found 384.0216; $[\alpha]_{\mathrm{D}}+22.2^{\circ}(\mathrm{c}=0.10, \mathrm{MeOH})$.

\section{References:}

1. Baker, S.R.; Clissold, D.W.; McKillop, A. Tetrahedron Lett., 1988, 29, 991-994. 2. Paquette, L.A.; Zeng, Q.; Tsui, H.; Johnston, J.N. J. Org. Chem., 1998, 63, 84918509 . 